\title{
COMMENTARY
}

\section{Controlling avian flu at the source}

\author{
Global agricultural authorities should harmonize with the public-health sector to ensure the exchange of flu \\ virus samples, and establish a single international standard for vaccines, say Robert Webster and Diane Hulse.
}

W e know that a flu pandemic will occur, but not when. Already in Asia the $\mathrm{H} 5 \mathrm{~N} 1$ avian flu virus has infected at least 88 people, killing 51 of them. For these outbreaks to become a pandemic, the only ingredient lacking is consistent human-to-human transmission. We do not know if the $\mathrm{H} 5 \mathrm{~N} 1$ vector has this capability if it does, the effects will be catastrophic.

Certain aspects of pandemic planning have been well considered. But a global strategy for preventing pandemics at their source - in the animals, mostly poultry, that carry the virus has received relatively little attention. Here we highlight two examples of successful intervention strategies for controlling $\mathrm{H} 5 \mathrm{~N} 1$ transmission to humans, which we believe merit greater consideration.

In the first documented instance of bird-tohuman infection with the H5N1 flu virus in 1997 , Hong Kong reacted by destroying its entire poultry population of 1.5 million birds within three days. Many believe that this action averted a pandemic by immediately removing opportunities for further human exposure. In addition to culling, Hong Kong introduced better market conditions, sanitation and vaccination. More recently, Thailand has been successful with the time-honoured agricultural practice of surveillance, restriction of poultry movement, and culling, with farmer compensation.

Both these successes were achieved at high cost, in terms of the number of birds culled and money spent. But moves by international agencies should now help countries achieve the same results with fewer sacrifices.

\section{Access to virussamples}

Because of the role of animals in any human pandemic, the United Nations' Food and Agriculture Organization (FAO) and the World Organization for Animal Health (OIE) are involved in pandemic planning, together with the World Health Organization (WHO). The mission of these agencies is in many ways complimentary: the WHO protects human health, the OIE protects the health of animals, and the FAO is responsible for animal productivity. But when it comes to pandemic planning for flu, these agencies are not always in harmony.

For example, the lack of animal H5N1 flu viruses collected from the field for analysis is

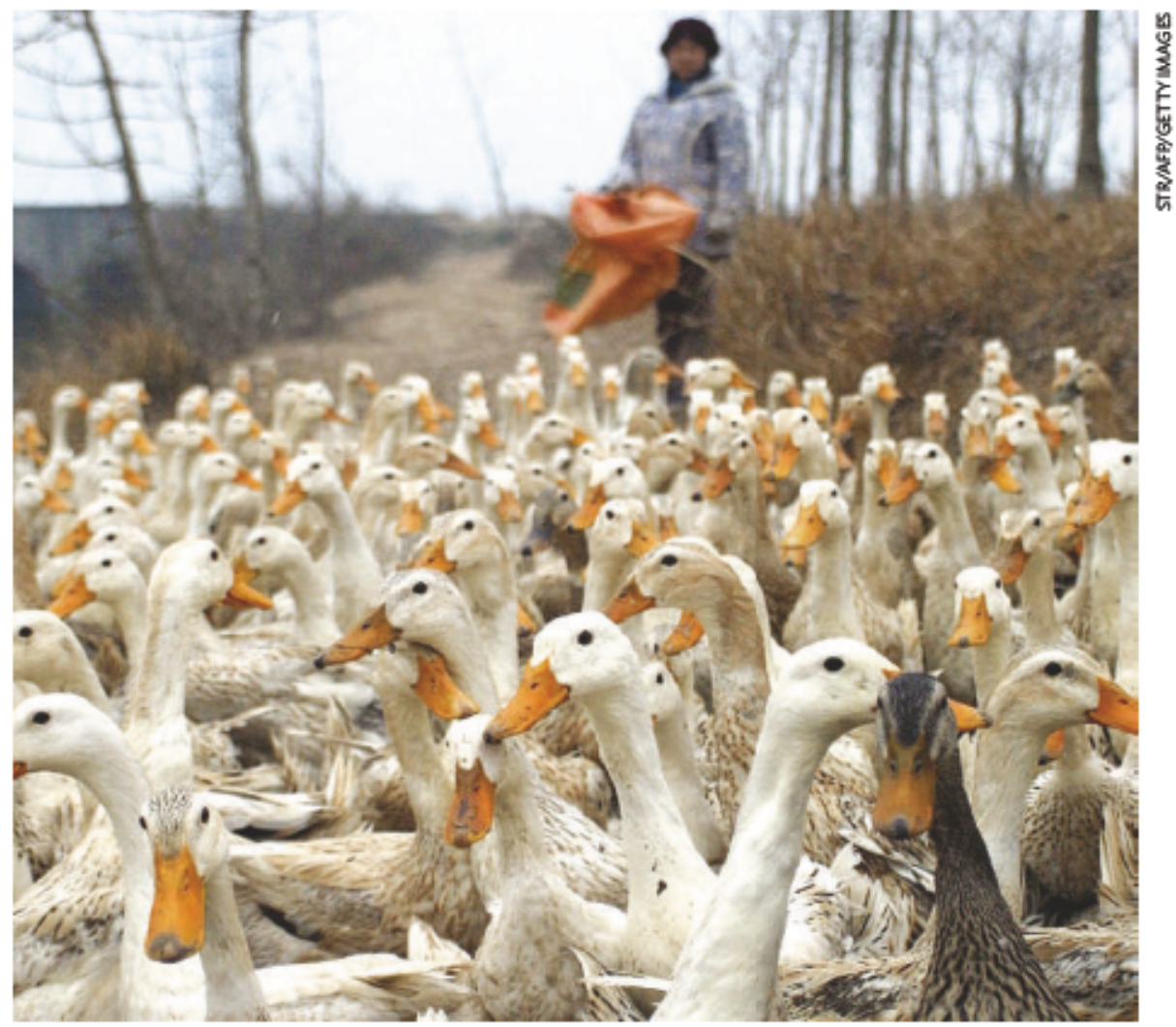

Ther e is now no doubt that the H5N1 avian flu virus is endemic in domestic ducks in Asia.

an area of concern (see Nature 435, 131; 2005). Such analyses are essential for vaccine design and for effective policy decisions. The exchange of animal $\mathrm{H} 5 \mathrm{~N} 1$ flu viruses between countries and researchers is hampered by free-trade embargoes, national pride, intellectual property, lack of political will, biosafety and bioterrorism considerations, and inadequate infrastructure. There are no simple answers to these and many other difficulties. But such problems are largely man-made, and so should be resolvable.

One of the aftermaths of the severe acute respiratory syndrome (SARS) epidemic is a raised profile of laboratory accidents as sources of disease. Countries with inadequate infrastructure to work with $\mathrm{H} 5 \mathrm{~N} 1$ influenza are now advised to use molecular probes to detect virulent strains, and not to attempt to isolate $\mathrm{H} 5 \mathrm{~N} 1$ viruses, which requires biosafety level 3 (BSL3) facilities. Although this seems like sound advice, others may argue for international provision of mobile BSL3 laboratories, trained staff, and assistance to build up local infrastructure. The FAO has been working diligently in this respect, but the funding is running out. This is one of many reasons why there are so few $\mathrm{H} 5 \mathrm{~N} 1$ virus samples from humans and other animals

\section{Setting an example}

H5N1 bird flu is not a new threat. The 1997 Hong Kong outbreak killed 6 of 18 infected people ${ }^{1}$. Indeed, the situation there today owes much to the response since then. Although no H5N1 flu viruses have been isolated from domestic poultry or humans in Hong Kong since 2004, the virus has been circulating in herons and falcons. It is also notable that the H5N1 virus has continued to evolve: in late 2002 it acquired the ability to kill its natural host, wild waterfowl, and spread across ten countries in southeast Asia ${ }^{2}$. The virus has also expanded its host range to include tigers and domestic cats ${ }^{3}$. The concern is now whether 


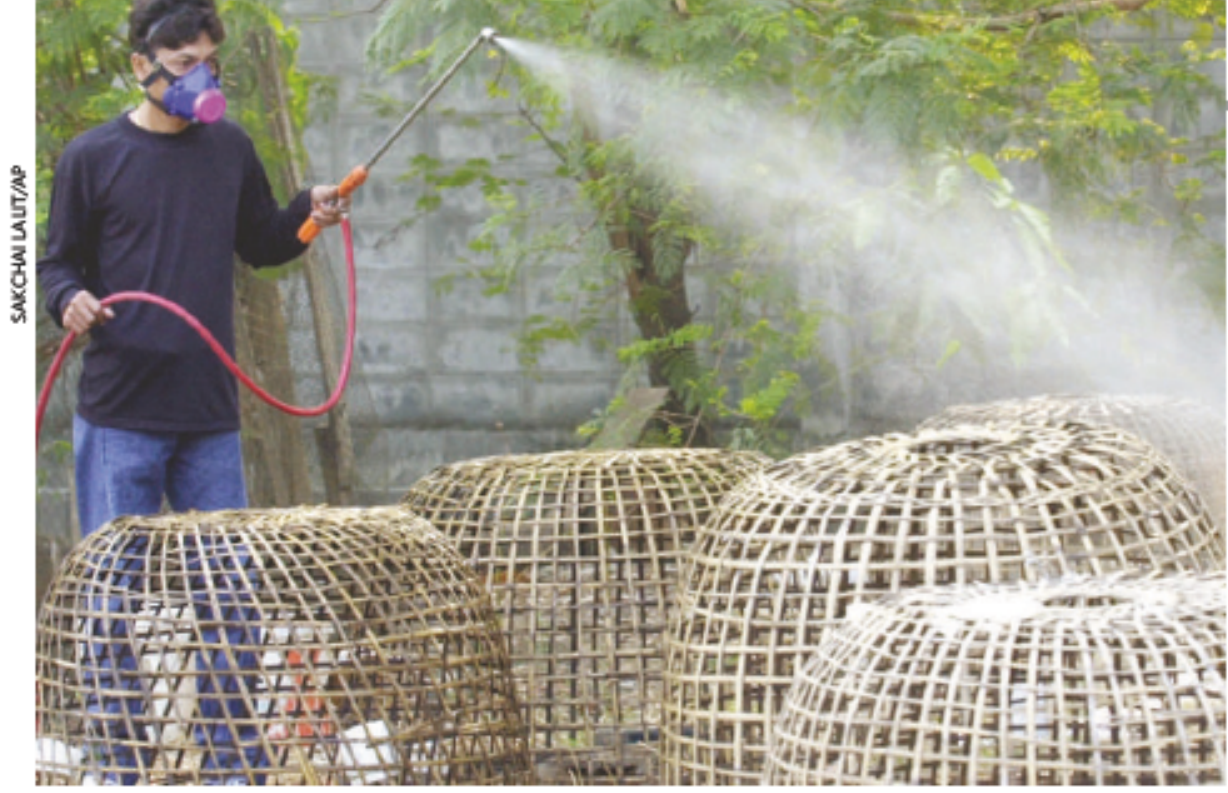

Even simple measures to curb infection, such as cleaning poultry cages, are not yet widely used.

it will acquire consistent human-to-human transmission.

So what has Hong Kong done to successfully control H5N1 bird flu? The steps taken include: modifying live-poultry marketing practices by banning ducks, geese and quail (the original sources of flu); introducing two 'dean days' per month, when all markets are simultaneously emptied and cleaned; vaccinating all locally raised and imported poultry with an inactivated $\mathrm{H} 5 \mathrm{~N} 1$ vaccine; and introducing unvaccinated 'sentinel' poultry into each chicken house. These goals have been achieved by model interagency cooperation between the Hong Kong departments of health, agriculture, and food and safety, together with the University of Hong Kong. But why has the Hong Kong model apparently been ignored elsewhere?

The easy answer is that only Hong Kong can afford such rigorous control; the approach is too costly for other, larger countries. But although clean days and improvements to sanitation are disruptive, both are relatively inexpensive. Even simple changes, such as the separation of ducks and chickens in live poultry markets, have not been strongly advocated by international agencies. The question of vaccination is more complex, largely because agricultural vaccines vary in quality.

Although both are efficacious, the flu vaccines for humans and for chickens meet different standards. The few comparative tests that have been done on agricultural vaccines from different suppliers show that some are good and some are bad. Bad vaccines prevent the symptoms of disease but not virus excretion, which can lead to later infection. One of the many arguments against the use of agricultural vaccines is that they promote the selection of mutations in the circulating virus, perpetuating the risk of infection either in the original species or in others. This effect has been documented recently for the $\mathrm{H} 5 \mathrm{~N} 2$ vaccines used in Central America ${ }^{4}$. However, mutations and selection will occur whether vaccines are used or not. In Hong Kong, sentinel birds provide a way to monitor virus excretion and the generation of mutations.

We propose that global agricultural authorities harmonize with the human public-health sector to establish a single international standard for a vaccine that is based on antigen content. The current dogma is that agricultural vaccines do not produce sufficient immunity to completely prevent transmission, making culling the preferred alternative. But it may be that high-quality vaccine strains matched to the circulating strains can reduce the level of circulating virus below the transmissible level. The technology for producing inexpensive agricultural vaccines using reverse genetics is available and should be developed.

\section{Harmonizing vaccine use}

Arguments about culling and vaccination arise particularly in Thailand and Vietnam. There is now no doubt that highly pathogenic $\mathrm{H} 5 \mathrm{~N} 1$ avian influenza virus is endemic in domestic ducks in Asia. In Thailand and Vietnam, domestic duck raising increases the likelihood of H5N1 infection by up to eightfold. But the role of ducks in the continuing spread of $\mathrm{H} 5 \mathrm{~N} 1$ is complicated because some virus strains kill ducks whereas others are benign in ducks but lethal for chickens and probably humans.

Since December 2004,41 people have been infected with $\mathrm{H} 5 \mathrm{~N} 1$ in Vietnam and 16 have died, but none have been infected in Thailand. Why this difference? Thailand has pursued a massive surveillance of poultry in its 'X-ray programme'; trained personnel visited all villages in late 2004 to collect virological and serological samples. The culling of ducks in Thailand (with farmer compensation) has reduced the flocks of ducks that were positive for $\mathrm{H} 5 \mathrm{~N} 1$ from almost $40 \%$ infected in October 2004 to almost undetectable levels in March 2005. Thus, reducing H5N1 infection in poultry clearly reduces the threat to humans.

Could vaccinating ducks have similar benefits to culling? Duck vaccines for flu are in an even worse state than chicken vaccines, virtu- ally nothing is known. Unless high-quality vaccines are developed and used, H5N1 viruses that are benign in ducks but lethal for chickens and humans will be selected (although this is likely to occur naturally anyway). Our aim must be to reduce the virus load in ducks below the transmissible level, as was achieved by vaccinating chickens in Hong Kong. This is probably the approach that has the best chance of reducing the inevitability of $\mathrm{H} 5 \mathrm{~N} 1$ acquiring consistent human-to-human transmission.

Thai authorities know that even if $\mathrm{H} 5 \mathrm{~N} 1$ is eradicated from domestic poultry this year, the virus will probably return from neighbouring countries. Also, it is not known whether highly pathogenic $\mathrm{H} 5 \mathrm{~N} 1$ variants are endemic in the wild waterfowl that breed in Siberia and migrate through the region. Flu, like SARS, knows no borders, and it is likely that H5N1 will continue to cross them. Consequently, relying on culling alone is a risky strategy.

China, which does not export much poultry, adopted the generalized use of poultry vaccines to control the H5N1 epidemic in 2004, and has reported no clinical outbreaks in domestic poultry since. Indonesia has also used poultry vaccines, although there have been reported cases of $\mathrm{H} 5 \mathrm{~N} 1$ in poultry and pigs this year. Vietnam has recently decided to start testing poultry vaccines this summer.

Thailand is a major poultry-exporting country. And its trading partners would prefer it not to use poultry vaccines, which can mask residual $\mathrm{H} 5 \mathrm{~N} 1$ viruses. However, Thailand is now investigating the use of flu vaccines for backyard poultry, free-range ducks and fighting cocks (not commercial poultry). This decision represents a major shift in policy. The use of $\mathrm{H} 5 \mathrm{~N} 1$ vaccines for such 'open range' poultry is a prudent step, and should be encouraged in other countries in the region.

Commercial poultry and backyard poultry are currently considered to be linked. Therefore during an outbreak all birds are culled, which is expensive. The OIE is now considering rule changes that would recognize different categories of poultry (closed chicken farms and backyard poultry). Countries could then move to having a system of zones based on poultry populations with different disease status. In this way, they could develop disease-free zones, perhaps by the selective use of vaccines, and recover some export capacity. We hope that the OIE's initiative indicates growing harmony among international agencies.

Robert Webster and Diane Hulse are in the Department of Infectious Diseases, St Jude Children's Research Hospital, Division of Virology, Memphis, Tennessee 38105, USA.

1. de Jong, I C, Clas, E.C., Osterhaus, A.D., Webstex, R. G. \&L Lim, W L Nature 389, 554 (1997).

2. Li,K.S.etal. Nature 430, 209-213(2004).

3. Kuiken, T.et al. Science 306, 241 (2004).

4. Lee, C.W. etal. J. Viral. 78, 8372-8381 (2004). 